\title{
Capsular Contracture after Breast Augmentation: An Update for Clinical Practice
}

\author{
Hannah Headon ${ }^{1}$, Adbul Kasem ${ }^{2}$, Kefah Mokbel ${ }^{3}$ \\ ${ }^{1}$ King's College London Medical School, London; ${ }^{2}$ Oncoplastic Breast Surgeon Medway NHS Trust, Kent; ${ }^{3}$ Aesthetic and Reconstructive \\ Breast Surgery, The London Breast Institute, London, UK
}

Capsular contracture is the most common complication following implant based breast surgery and is one of the most common reasons for reoperation. Therefore, it is important to try and understand why this happens, and what can be done to reduce its incidence. A literature search using the MEDLINE database was conducted including search terms 'capsular contracture breast augmentation', 'capsular contracture pathogenesis', 'capsular contracture incidence', and 'capsular contracture management', which yielded 82 results which met inclusion criteria. Capsular contracture is caused by an excessive fibrotic reaction to a foreign body (the implant) and has an overall incidence of 10.6\%. Risk factors that were identified included the use of smooth (vs. textured) implants, a subglandular (vs. submuscular) placement, use of a silicone (vs. saline) filled implant and previous radiotherapy to the breast. The standard management of capsular contracture is surgical via a capsulectomy or capsulotomy. Medical treatment using the off-label leukotriene receptor antagonist Zafirlukast has been reported to reduce severity and help prevent capsular contracture from forming, as has the use of acellular dermal matrices, botox and neopocket formation. However, nearly all therapeutic approaches are associated with a significant rate of recurrence. Capsular contracture is a multifactorial fibrotic process the precise cause of which is still unknown. The incidence of contracture developing is lower with the use of textured implants, submuscular placement and the use of polyurethane coated implants. Symptomatic capsular contracture is usually managed surgically, however recent research has focussed on preventing capsular contracture from occurring, or treating it with autologous fat transfer.

Keywords Capsular contracture / Breast implants / Mammoplasty
Correspondence: Kefah Mokbel Breast Surgery, The London Breast Institute, Princess Grace Hospital, 45 Nottingham Place, London W1U $5 N Y$, UK

Tel: +44-207-908-2040

Fax: +44-207-908-2275

E-mail: kefahmokbel@hotmail.com

Sir John Swire Foundation funded the research.

No potential conflict of interest relevant to this article was reported.

\section{INTRODUCTION AND BACKGROUND}

Breast augmentation is one of the most commonly performed cosmetic procedures [1]. This commonly involves an implant being inserted within the breast in order to alter the shape and size of the breast. Various types of implant are available and they are generally divided according to the shape of the implant (whether rounded or anatomically shaped), or the surface texture (whether smooth or textured) or the gel used to fill the implant (usually either saline or silicone) [2]. Silicone breast implants are currently the most popular and accepted material for use in breast augmentation. These can take one of two forms: a silicone outer shell with a silicone gel filling, or a silicone outer shell with 
an alternative filling, such as saline [3]. However, the general consensus is that the postsurgical mechanical behaviour of silicone implants is more like natural breast tissue when compared to saline [4].

As with any surgery, implant based breast augmentation has been associated with a number of risks and complications. The most common complication is capsular contracture as identified in a 25 years longitudinal study by Handel et al. [5], with implant rupture and malposition also being cited as reasons for reoperation. Introduction of non-biologic materials into the body always induces formation of a capsule, but in the breast this may be particularly severe. Capsular contracture is a local complication thought to occur due to an excessive fibrotic foreign body reaction to the implant. It is thought to be an inflammatory reaction which causes fibrosis through the production of collagen [3], leading to excessively firm and painful breasts [6]. If severe enough, this can require reoperation [7]. For an example of how capsular contracture may present (Fig. 1).

\section{Fig. 1. Grade IV capsular contracture}

This is a picture of a 40-year-old woman who has Baker grade IV capsular contracture. On the left, the breast is noticeably higher and there is noticeable skin puckering along the medial side. The nipple is higher relative to the right side.

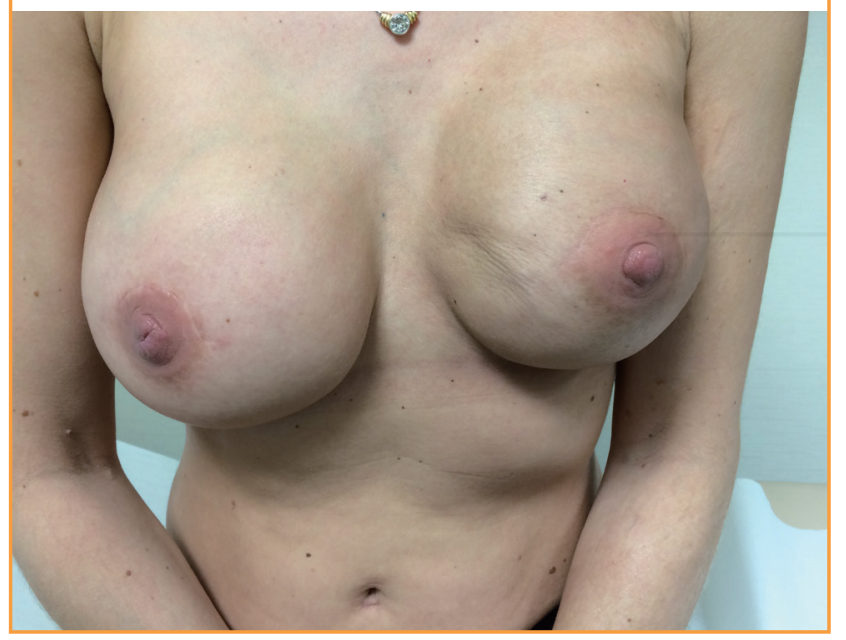

Capsular contracture is traditionally classified using the Baker classification system, a subjective classification system that is based upon clinical findings in the patient by the physician. It is divided into four classes: I and II are not clinically significant, in that I describes a breast that looks and feels absolutely natural and II describes a breast with minimal contracture in that the surgeon can tell surgery has been performed but there are no symptoms. Class III and IV are clinically significant and symptomatic, with III describing moderate contracture with some firmness felt by the patient and IV describing severe contracture which is obvious from observation and symptomatic in the patient [8].

Individual studies have published incidence rates of capsular contracture ranging from $2.8 \%$ to $20.4 \%$ [9-14]. A recent systematic review published a combined overall rate of $3.6 \%$ following augmentation surgery [2]. However, there is a wide range of heterogeneity between studies in terms of follow up times which may affect capsular contracture development rates, as well as a lack of standardisation in the type of implant and surgical techniques used. The individual study variables are outlined in Table 1. Despite the wide range of incidence rates reported, it is widely accepted that capsular contracture is the most common complication following implant based breast surgery. Despite this, the aetiology and pathogenesis is not yet clear, although it appears to be multifactorial. In order to effectively treat, or even prevent capsular contracture, it is important to understand the mechanisms that lead to it.

The aim of this paper is to review the current literature available in order to look at the pathogenesis, the risk factors and the optimal management of capsular contracture following breast augmentation surgery.

\section{PATHOGENESIS OF CAPSULAR CONTRACTURE}

The pathogenesis of capsular contracture is thought to be multifactorial. It is known that capsular contracture is essentially an excessive fibrotic foreign body reaction that occurs after implan-

Table 1. A table showing the individually reported incidence rates for capsular contracture following implant based breast augmentation surgery

\begin{tabular}{|c|c|c|c|c|}
\hline Study name & Type of implant & No. of participants & $\begin{array}{l}\text { Average follow up } \\
\text { time (yr) }\end{array}$ & $\begin{array}{c}\text { Incidence of capsular } \\
\text { contracture }(\%)\end{array}$ \\
\hline Spear et al. [9], 2014 & Natrelle, round silicone & 715 & 6 & 18.9 \\
\hline Blount et al. [10], 2013 & Various & 856 & $14.9 \mathrm{mo}$ & 2.8 \\
\hline Stutman et al. [11], 2012 & Various & 619 & 2.4 & 7.6 \\
\hline Codner et al. [12], 2011 & Various & 812 & 6 & 8.2 \\
\hline Sevin et al. [13], 2006 & Textured silicone gel round implants (McGhan) & 210 & 8 & 8 \\
\hline Gutowski et al. [14], 1997 & Saline filled implants & 504 & 6 & 20.4 \\
\hline Overall & Various & 3,716 & $1.24-8$ & 10.6 \\
\hline
\end{tabular}


tation $[6,15]$. Whist on the one hand, this fibrotic reaction helps to maintain the position of the implant, if excessive it can lead to pain and deformity of the breast [16].

The cellular composition of the capsule has been studied extensively, and the results seem to suggest a role of the immune system in the pathogenesis of capsular contracture. Macrophages, lymphocytes and fibroblasts have been reported to be the predominant cell types within the capsule. Fibroblasts accumulate at the 'contact zone' of the implant and the capsule $[6,17,18]$, and it has been reported that the number of fibroblasts within the capsule correlates with the Baker grade, with an increased number found in grade IV capsular contracture when compared to grade I [19]. As fibroblasts produce collagen, they have been linked to the initial formation of the capsule. Histological evaluation of capsular tissue has shown that the majority of the tissue is made up of uniformly distributed collagen fibres $[3,19]$. The orientation and organisation of the collagen fibres appears to change as contracture severity worsens: the fibres become thicker and establish themselves in cable-like bundles which orientate themselves perpendicular to the fibroblasts to produce a helical orientation as the severity increases [20]. The fibroblasts in turn orientate themselves planarly when studied in capsules surrounding smooth implants [16].

Mast cells have also been investigated with regards to their involvement in the pathogenesis of capsular contracture. A study of capsular tissue taken from patients who required revision surgery or removal of expander found that mast cells within the capsular tissue expressed renin, histamine and tumour growth factor $\beta$-1 (TGF- $\beta$ ) [19]. Additionally, capsular fibroblasts express corresponding receptors, indicating that the mast cells may activate neighbouring fibroblasts via a paracrine pathway following mast cell degranulation, leading to increased production of collagen and therefore development of capsule contracture.

The role of myofibroblasts in the development of capsule contracture has also been investigated. Myofibroblasts are contractile fibroblasts which are thought to provide a contractile force which decreases the surface area of the capsule whilst the collagen matrix remodels and stabilises the contracture. Interestingly, these cells have been found to express oestrogen receptor- $\alpha$ (ER$\alpha)$ and/or $\beta$ (ER- $\beta$ ), which are acted on by $17-\beta$-oestrodiol to increase their contractile force [21]. In the same study, Persichetti et al. [21] investigated the effect of anti-oestrogenic therapy through administration of Tamoxifen. In the treatment group, there was decreased TGF- $\beta$ production and myofibroblast contraction, indicating a role of the oestrogen hormones. Therefore, anti-oestrogenic therapy may help reduce the severity or perhaps prevent capsular contraction from developing.

A role of T cells has also been hypothesised. CD4+ T cells have been found to be involved [22-25], with one study finding twice the level of CD4+ cells in those with symptomatic capsular contracture than those who were asymptomatic asymptomatic [16], and others showing an increased response in peripheral blood monocytes in response to silicon [24]. It is thought that the CD4+ cells may then produce a specific profibrotic cytokine profile, mediating an immune response by activating $\mathrm{TH} 1$ and $\mathrm{TH} 17$ cells [16].

Despite knowing the cells that appear to involved in the process of capsular contracture, it is important to know the way in which they are activated in order to understand how exactly it develops and thus develop strategies to try and prevent it. As well as TGF- $\beta$, other molecules that have been associated include tumour necrosis factor- $\alpha$ (TNF- $\alpha$ ), matrix metalloproteinase 2 (MMP-2) and its endogenous inhibitors, tissue inhibitors of metalloproteinases 1 and 2 (TIMP- 1 and TIMP-2). TNF- $\alpha$ has been reported to be expressed on fibroblasts, macrophages and extracellularly close to the prosthesis, and its presence is associated with an increased grade of Baker contracture [17]. Similarly, MMP-2 expression is significantly increased with more severe grades of Baker classification, with a reduced ratio of MMP to TIMP, a similar scenario as observed in other progressive fibrotic disorders [26].

Currently, the majority of the literature focuses on the role of TGF- $\beta$. A recent study by Katzel et al. [27] indicated that TGF- $\beta$ may act as a 'master switch', allowing a cascade of reactions which subsequently leads to capsular contracture. TGF- $\beta$ signals through the phosphorylation of Smad-3. In mice with a knock out of this signalling pathway, capsules were thinner and more regular after being exposed to radiotherapy when compared to controls, which showed thicker capsules made up or irregular collagen. As mentioned previously, women treated with Tamoxifen produced less TGF- $\beta$ and also had a lower incidence of grade III-IV capsular contracture than those who were not treated [21]. This may be due to a reduction in oestrogen signalling in myofibroblasts, however may also be due to a reduction in activation of fibroblasts by TGF- $\beta[28,29]$. Other immunological agents have also been reported as having a role in the development of capsular contracture. These include connective tissue growth factor factor and interleukins 4, 6, 10, 13, and 21 amongst others, all of which promote fibrosis [30]. However, more research on their individual roles needs to be conducted before their influence can be fully evaluated. Interleukin- 8 has been suggested as a potential biomarker after Kyle et al. [31] reported that it was upregulated along with matrix metallopeptidase 12 . This is not the only molecule that has been discussed as a potential biomarker. Wolfram et al. [32] also found that, in those with silicone breast implants, circulating immune complexes, antipolymer antibodies and solu- 
ble intercellular adhesion molecule-1 were also upregulated, and their levels correlated with the development of fibrosis.

Although the majority of current evidence appears to support the role of the immune system, historically it has been suggested that capsular contracture may result from an exaggerated inflammatory response caused by a haematoma or infection. A number of studies have found that the presence of bacteria accelerates contracture development in animal models $[33,34]$. In particular, the role of coagulase negative staphylococcus species has been investigated with some interest, with a lot of focus on involvement of Staphylococcus epidermidis, where its presence on implants in animal models has been found to cause increased capsule pressure and a thicker capsule that has an increased density of collagen and increased angiogenesis, all of which are subsequently associated with capsular contracture [33]. However, this theory fails to take into account a number of variables. Firstly, capsular contracture does not affect every patient; therefore it is likely that phenotype plays a part in the reaction. Also, this theory fails to take into account the time lapse often present between surgery and clinically significant contracture development, which can vary greatly. Additionally, prophylactic strategies such as antibiotic irrigation have failed to show any difference in capsular contracture rates, with reports that washing the implants antispetcially or giving systemic antibiotics having only minor effects, if any [16]. In one study, triple antibiotic breast irrigation was found not to be associated with a significant reduction in the severity of capsular contracture, with rates published at $3.7 \%$ and $3.6 \%$ with and without antibiotic irrigation respectively [35], indicating that biofilm formation may be an incidental finding, rather than a cause and effect in the setting of capsular contracture. Additionally, in a porcine experiment, animals which were given implants and inoculated with S. epidermidis did show an increase in the incidence of capsular contracture when compared to those not inoculated, however, there were still incidences of capsular contracture in the non-inoculated group [34]. This indicates that infection, and the subsequent inflammatory response, whilst maybe increasing the rate at which capsular contracture develops does not in fact cause it.

\section{STRATEGIES TO DECREASE THE INCIDENCE OF CAPSULAR CONTRACTURE}

\section{Implant choice}

There is a wide variety of implants that are available for implant based breast surgery, each of which have slightly different properties. These can be broadly separated into whether the surface of the implant is smooth or textured, or whether they are filled with silicone gel or saline and whether the implants are rounded or anatomically shaped. A number of companies provide and develop breast implants, two of the most widely used being Allergan (Allergan Inc., Los Angeles, CA, USA) and Mentor (Mentor Worldwide LLC., Santa Barabara, CA, USA).

Patients tend to be consulted on the type of implant they would prefer, be that silicone based or saline based, so therefore it is important to understand the risks associated with each in order to allow fully informed consent. Silicone gel implants, first introduced in 1961, currently dominate the world market and this is reflected in the much larger range available for use [36]. In the past, there were concerns with the use of silicone gel implants, especially in the United States of America where their use was restricted to clinical trials up until 2006 due to safety concerns. However, long term studies assessing their safety are currently underway. In terms of the Allergan models, there is both rounded and shaped implants available. A long term multicentre clinical trial is currently underway assessing the Natrelle Style 410 implant, a form stable highly cohesive shaped model. Results from 3 years reported a capsular contracture rate of $4.8 \%$ in primary augmentation patients [37], with results at 6 years echoing this with a reported rate of $4.6 \%$ in the augmentation cohort [38]. A further study reported a 5.6\% incidence of capsular contracture [39]. On the other hand, an ongoing study looking at the use of Mentor round silicone MemoryGel implants in 1,007 women found that the risk of Baker grade III/IV at three years was $8.1 \%$ in primary augmentation patients [40]. A similar study looking at shaped Mentor silicone implants reported a 2.4\% risk of Baker grade III/IV capsular contracture [41]. Therefore, both suppliers have acceptably low capsular contracture rates with silicone models and the long term results of these multicentre trials are likely to have interesting findings. Silicone as a filler material has a number of advantages: it is thought that the implants act more like natural breast tissue and due to extensive development of silicone implants are more technologically innovated. However, saline is still an option for patients.

Saline implants also offer a number of advantages. The main advantage is that saline implants sidestep concerns regarding rupture of silicone implants. If saline implants rupture, the fluid is absorbed harmlessly into the body, however exposure to silicone gel is thought to be more dangerous [36]. There is less variety available with saline implants and they have remained relatively unchanged over the last few years. In terms of capsular contracture risk, a ten year prospective study of the Allergan Natrelle saline filled implants found a $20.8 \%$ capsular contracture incidence in breast augmentation cases [42], indicating a much a higher risk than with silicone implants. This is in contract to a meta-analysis carried out in 2008 which reported a greater than 
two-fold increased risk of capsular contracture following treatment with silicone gel implants as opposed to saline. However, these results were limited by the poor scientific qualities of the studies involved and a high variability of the different types of implant used in the various studies [43]. Ideally, more research comparing long term use of saline and silicone implants would be useful or an updated meta-analysis which includes the results of the studies mentioned above in order to evaluate any potential difference in capsular contracture risk.

The surface texture of the implant has also been found to have an effect on the incidence of capsular contracture. In terms of the surface of the implant, it has been established that textured implants are associated with a lower risk of clinically significant capsular contracture.as reported by a number of meta-analyses [44-46]. Textured implants were introduced following the observation that implants covered with polyurethane resulted in a more textured surface, and these had a lower incidence of capsular contracture [47]. A randomised controlled trial found that, with submuscular placement of the implant, patients had reduced symptoms of contracture when a textured implant was used [48]. This finding was supported by a recent risk analysis, which concluded that smooth implants resulted in increased odds of capsular contracture [49]. A systematic review concurred, showing that using textured implants resulted in the number needed to treat was 8.8 and 6.7 for round and shaped implants respectively in order to prevent one incidence of Baker grade III-IV capsular contracture over 10 years [2]. Implants with a macrotextured surface were found to significantly decrease the risk of capsule contracture in particular, with participants in one particular study finding that when such implants were used, the capsule mimicked the feeling of natural breast firmness [50]. Recent research has attempted to link these findings back to pathogeneic hypotheses: one recent study found that, in comparison, textured implants were more likely to result in biofilm formation [51]. However, the overall higher incidence rate found with smooth implants suggests that biofilm production may not in fact play a large part in capsular contracture development.

Textured implants may be associated with a lower incidence of capsular contracture because of the way in which they interact with the surrounding tissue. The textured surface is thought to disrupt the contractile forces around the implant [16]. Animal studies have found that use of smooth implants led to thicker capsules with an increased concentration of collagen fibres, and additionally had a higher concentration of thick fibres [52]. It has been suggested that macrotextured implants lead to the formation of vectors of different lengths and directions, which in turn leads to a more natural breast texture [50]. However, it may appear that aggressively textured implants may be associated with other problems, with one study finding a link between the use of textured implants and double capsule formation, which was not seen with smooth implant use [53]. A more thorough understanding of the pathogenesis and development of capsular contracture at the molecular level would lead to an understanding of how implants interact with the surrounding tissue and therefore help to evaluate the best option in terms of choice of implant.

Polyurethane coated breast implants provide a textured outer surface and have been researched recently due to the reported associated low capsular contracture incidence rates. A recent study by Pompei et al. [54] looked at their use following radiotherapy, a recognised risk factor associated with capsular contracture. They found that capsular contracture occurred in $6.3 \%$ of patients who used polyurethane coated implants whereas those who had expanded textured implants inserted reported a rate of $21.7 \%$. Older studies have also found low incidences of capsular contracture. One study looking at their use in breast augmentation found that the incidence of capsular contracture was just $1 \%$ [55]. The authors hypothesised that this low rate was due to breakdown of the polyurethane coating causing remnants of the material to be retained within the capsule causing the collagen fibres to interweave. Histological examination has also found that in capsules surrounding polyurethane implants, there is less fibrotic tissue and less type 3 collagen than seen with textured implants [56].

\section{Surgical technique}

Decisions regarding the procedure have been shown to have an effect on the incidence of capsular contracture. It is commonly accepted that a submuscular placement, where the implant is placed behind the pectoralis major muscle, leads to a lower incidence of capsular contracture than when the implant is placed just under the skin, in a subglandular placement subglandular $[14,49,57]$. However, both placements can still lead to contraction. Siggelkow et al. [3] reported that $21 \%$ of patients with submuscular placement had an increase in Baker grading whereas in those with subglandular placement, $84 \%$ noticed an increase. A recent systematic review found that overall rates of capsular contracture with subglandular placement stood at $8.6 \%$, whereas for submuscular placement it was just $2.8 \%$ [2]. However, these findings are not universal, with some studies finding no difference between anatomical pocket location and rates of capsule contracture [12]. This suggests that a submuscular placement should be favoured over a subglandular placement in order to reduce future complications. Despite this, a meta-analysis incorporating all the most recent data is required in order to fully evaluate the impact location has on the development of capsule contracture when taking into account more modern implant models. 


\section{MANAGEMENT AND PREVENTION OF CAPSULAR CONTRACTURE: NEW STRATEGIES}

\section{Surgical methods}

Traditionally, capsular contracture is treated surgically, although it is important to note that treatment is only indicated in grades III and IV capsular contracture. The 'gold standard' treatment has been cited as being a capsulectomy with or without a capsulotomy [58] with one review finding that these procedures were performed in $3.2 \%$ of cases [2]. Two studies found that capsular contraction was the most common reason for reoperation when looking at the safety and efficacy of specific implant models [9,59]. Although capsulotomy has been found to be an effective surgical treatment for capsular contraction, it tends to recur so therefore multiple procedures may be needed in order to maintain soft breasts [60]. In fact, surgical treatment is associated with a significant risk of contracture recurrence approaching $25 \%$ in the first year, which may lead to a cascade of procedures [61]. The most common surgical treatment has been reported to be a total capsulectomy with a site change when indicated [62], however when the contracture has occurred in the submuscular plane this can be difficult and may lead to more complications [63].

A newer surgical management technique has been proposed which involves the formation of a so-called neopocket in which to place the implant. This involves the creation of a new subpectoral plane deep to the pectoralis major muscle but superficial to the anterior capsule, which is left intact to avoid further tissue damage. This allows use of the existing capsule and is usually done through an inframammary incision [64]. A retrospective review of 198 patients, $69.7 \%$ of which presented with capsular contracture, who were treated using this technique found a high success rate in reduction of contracture [65]. A further study by Castello et al. [66] concluded that neopocket formation was an effective one stage solution for the correction of augmentation induced deformities, and that complications remained completely resolved at 24.1 months follow-up. This could be a potential new standard of treatment for capsular contracture as allows the use of the existing capsule, but gives a new vascularised pocket in which to insert a new textured implant. However, at present this is only feasible in submuscular placement, as there is enough tissue to allow a new plane to be created.

Other surgical methods which have been discussed as treatment strategies for capsular contracture include the use of autologous fat transfer. This can take one of two forms: lipofilling in order to try and treat capsular contracture or a fat graft placed in the initial procedure along with a half sized implant. There have been some studies that have looked at dual autologous fat trans- fer and implant surgery in reconstruction cases which have reported favourable results. Salgarello et al. [67] found that when fat grafts were used 6 months after radiotherapy followed by implant placement 3 months later there was a reduced incidence of postoperative complications including capsular contracture at a 15 months follow-up. These results were echoed in a small study of 28 patients who had lipofilling 6 months after radiotherapy followed by implant placement 3 months later, there was no grade III/IV capsular contracture cases at a 17 months follow-up [68]. Although these cases have focussed on reconstruction cases, this is a technique that could be adapted for use in primary augmentation. It would be beneficial to have prospective cohort studies examining this procedure in augmentation cases with long term follow up in order to see if it had an effect on development of capsular contracture.

Additionally, there is recent evidence that suggests autologous fat transfer could be used to treat capsular contracture by increasing the vascularity of the tissue around the implant. In a study using pigs, they found that although there was no significant difference histologically or in Baker grading of the implants, fat injection did cause the capsule to soften in the treatment group, potentially due to neovascularisation in adjacent tissue [69]. They plan to follow this up in patients with mild to moderate capsule contracture in the future. Unfortunately this is the only study found within our literature search that addresses the use of lipofilling as a treatment of capsular contracture. Therefore, these results will have to be replicated before broad generalisations can be made. However, it does show promise for novel surgical treatments.

Furthermore, the use of acellular dermal matrices has been investigated as it is thought that these may alter the reactive processes at the tissue-implant interface, the so-called contact zone and therefore reduce the development of contracture [70-73]. In animal studies and small patient populations, this has been shown to have a positive effect. In one study in rats, it was found that the addition of an acellular dermal matrix placed around the implant led to a thinner myofibroblast layer at the contact zone and significantly decreased proliferation and inflammatory signs [71]. The authors hypothesised that this could lead to a reduction in contracture. Similarly, in a patient study which used acellular dermal matrices to prevent capsular contracture, no patients in the preventative group developed capsular contracture, leading them to conclude that its use significantly lowers the incidence of capsular contracture in the first 3.5 years after placement [73]. When used as a treatment for established capsular contracture, complete acellular dermal matrix coverage has been found to be an effective management strategy in grade III to IV capsular contracture, with no recurrence being seen at a follow 
up of 9.2 months, in comparison to earlier studies which used partial acellular dermal matrix coverage and found a recurrence of $6.3 \%$ [70]. A more recent long term study looked at 127 patients who had had a reconstruction with a porcine acellular dermal matrix and reported a capsular contracture incidence of just $0.6 \%$ at a mean follow up of 19.6 months, suggesting this may be an effective strategy at preventing or delaying the onset of capsular contracture [74]. Again, like with other proposed management strategies, long term studies are needed in a patient population in order to establish if acellular dermal matrices do in fact prevent capsular contracture, or if they simply delay its onset.

\section{Medicalmethods}

Although the exact eitopathogenesis of capsular contracture remains to be discerned, the inflammatory response seems to have a role. Therefore, it is thought that altering the inflammatory response through medication may reduce the incidence of capsular contracture. The leukotriene antagonist, Zafirlukast (AstroZeneca) has been used off label to investigate whether it has an effect on the development of contracture. Animal studies have found that when $5 \mathrm{mg} / \mathrm{kg}$ per day of Zafirlukast was injected around textured silicone implants, the capsules are thinner and more vascular with a lower collagen density $[75,76]$, indicating that the drug may be able to prevent capsular contracture from developing in the first place. A number of studies have also been carried out in women who have undergone implant based surgery. Generally speaking, although many of the studies have found a reduction in the Baker grading of capsular contraction with a dose of $20 \mathrm{mg}$ twice a day taken orally [77-81], however, there was no literature found in patients where Zafirlukast was used as a preventative measure, which may be useful to look at in the future. The most recent research involved a group of 60 patients with mild to severe capsular contracture who were prescribed Zafirlukast for six months. Mammary compliance was assessed before treatment and then monthly up to a year after cessation of treatment. Interestingly, whilst patients were actively taking treatment, contracture grading scores decreased however, on cessation of treatment, grading began to increase again [81], indicating that in order to have a long term effect patients would have to continue taking the drug, which could then lead to problems with side effects such as adverse effects on the liver. An earlier study suggested that the beneficial effects of Zafirlukast may be maintained for longer, finding that $73.1 \%$ of breasts either improved or maintained at 16.5 months after the initiation of therapy [77]. The majority of breasts in this study had mild contracture, with $52.8 \%$ of participants being graded 2 or under on a modified Baker grading scale, which may explain the more favourable results.
Botulinium toxin A has been reported to be effective at reducing keloid scarring. Implant capsules have been found to be histologically similar, so it has recently been investigated to see if it can reduce the amount of contracture or prevent its development. A study in rats which involved injected $0.5 \mathrm{~mL}$ ( 5 units) of botulinium toxin A into implant pockets reported that, in histological analysis of the capsule after 6 weeks, capsular thickness was reduced, there was a decreased number of inflammatory cells and decreased TGF- $\beta 1$ expression with a loose and well organised collagen pattern in the experimental group when compared to control, who were injected with $0.5 \mathrm{~mL}$ of saline [82]. A more recent study in mice concurred, also showing reduced expression of TGF- $\beta 1$ and reduced capsular thickness in treatment groups [83]. It has been suggested that botulinium toxin A may therefore reduce the development of capsular contracture by interrupting TGF- $\beta 1$ signalling, subsequently reducing the differentiation of fibroblasts to myofibroblasts. However, despite promising results, more research needs to be carried out before conclusions can be drawn, with a need to evaluate the use of it in patient populations.

Antiadhesion barrier solution (AABS) (Guardix, Hanmi Medical Co., Seoul, Korea) has also been studied with regards to its effect at reducing the development of capsular contracture. Animal studies have showed promising results. A study in rats that looked at the use of AABS and compared to a control group and to a fibrin treated group found that, in treatment groups, there were thinner capsules and significantly decreased amount of type 1 collagen compared to controls, but that there was no significant difference between the AABS group and the fibrin treated group [84]. Moreover, another group reported that the use of $0.1 \mathrm{~mL}$ AABS with silicone blocks in rates led to a significantly decreased inflammatory cell count and capsular thickness when compared to controls after 4 weeks [85]. Furthermore, a rabbit study which kept the implants in situ for 2 months following AABS treatment reported that, although there was no significant difference in capsule thickness or contracture on gross analysis, histologically the capsules were thinned and had a lower density of collagen and a lower ratio of myofibroblasts when compared to controls [86]. Although these results seem promising, there is currently no long term data, and clinical trials would need to be conducted in the future in order to evaluate if these results can be replicated in a patient population.

\section{ANAPLASTIC LARGE CELL LYMPHOMA}

Another risk associated with the use of implants is the possibility of anaplastic large cell lymphoma (ALCL). This is a rare type 
of non-Hodgkin's lymphoma and most cases occur in the capsule surrounding the implant and it is thought to be potentially associated with prolonged inflammatory states, similar to the theoretical pathogenesis of capsular contracture. A recent review concluded that there was a relative risk of 18.2 that ALCL would occur in women with breast implants compared to those without implants when looking at the 71 case studies published at the time, however the absolute risk remains low, ranging from $1: 500,000$ to $1: 3,000,000$ [87]. These figures are yet to be confirmed with further studies, and it is possible that they may change in light of updated numbers of case reports and increased global awareness of the disease. It is important to note that most patients who had disease confined to the implant capsule have a good prognosis, with the majority achieving complete remission [88].
For an example of the histological appearance of ALCL associated with implants (Fig. 2). The overall incidence is quite low, estimated to be 1 in 1 million per year [89], however women who develop this condition may present with capsular contracture and an effusion within the capsule. Alternatively, women may also present with an effusion and a mass lesion. Interestingly, those who present with effusion with or without capsular contracture have a better prognosis than those presenting with a mass [90]. Recurrent capsule contracture has been identified as a possible independent presenting symptom in those with ALCL, and possibly capsular contracture occurring years after the first surgery which appears to refractory to usual management techniques [91]. This should be borne in mind when assessing a patient with capsular contracture, as earlier treatment may lead to

Fig. 2. Atypical large cell lymphoma complicating augmentation mammoplasty

These are microphotographs showing the appearance of anaplastic large cell lymphoma in the capsule surrounding an implant. Most cases are of a B-cell phenotype. Pleomorphic cells with anaplastic nuclei and prominent nucleoli can be seen. The magnifications are as follows: (A) $\times 0.35$, (B) $\times 5.09$, (C) $\times 11.1$, and (D) $\times 28$.1. All micrographs show H\&E staining.
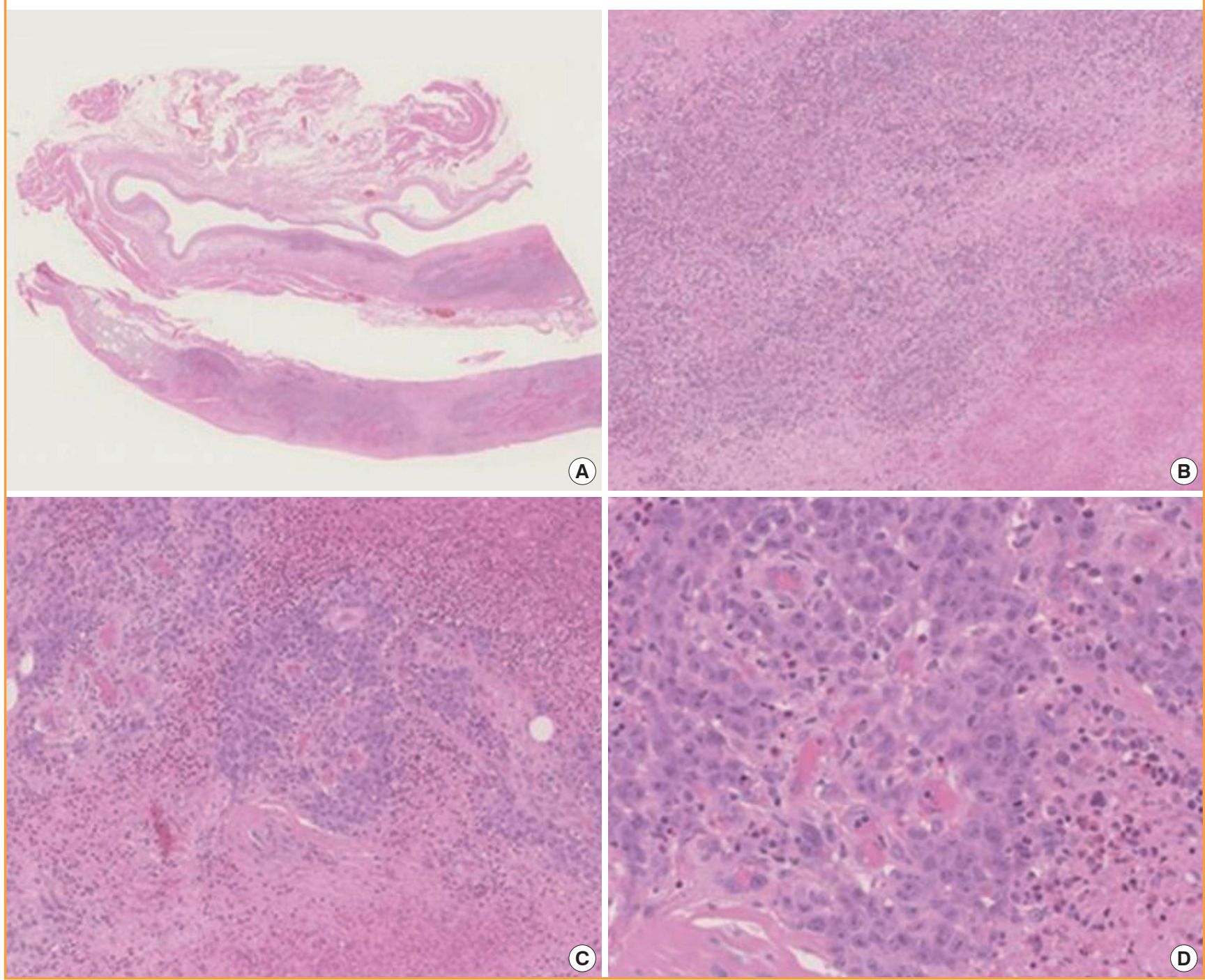
a better prognosis. However, more research on the link between capsular contracture and ALCL needs to be undertaken before definite conclusions can be made: ALCL is rare and not all women who develop capsule contracture will develop ALCL.

\section{CONCLUSIONS}

Capsular contracture is the most common complication following breast augmentation surgery. This literature review has attempted to outline some of the recent research regarding capsular contracture after breast augmentation surgery in order to identify risk factors and potential management strategies. From the current literature, it would appear that capsular contracture is a multifactorial process which involves inflammatory processes which then cause a fibrotic reaction in the tissue surrounding the implant, although the precise trigger and the reason why this happens is still not clear. Despite this, risk factors have been identified. From the currently available research, it appears that smooth implants, paced in a subglandular position have the biggest risk of developing capsular contracture.

Medical treatment of symptomatic cases seems to be ineffective and surgical therapy is associated with a significant rate of recurrence. However, there is a flurry of promising new research attempting to elucidate new management strategies or prophylactic strategies attempting to stop capsular contracture before it begins. Of these, the use of acellular dermal matrices has received the most attention, but new research looking at autologous fat transfer or using implants with lower incidence rates such as those coated in polyurethane shows promising results. More research in these areas needs to be undertaken in order to find the best possible method at avoiding reoperation and explantation.

The results of this review can add to current clinical practice by providing more information to women about the risks regarding breast augmentation surgery and help towards fully informed consent. In the future, it is recommended that a thorough meta-analysis is carried out which takes into account all the factors that can affect the incidence of capsular contracture: the anatomical placement of the implant, the surface texture of the implant and the core type of the implant as well as a history of radiotherapy to the breast. Reconstruction and radiotherapy have been identified as independent risk factors for capsular contracture, and more research should be carried out in order to establish the amount of risk conferred. In addition, new strategies for preventing and treating capsular contracture need to be evaluated in large patient population studies in order to evaluate their real life benefits. Due to findings that the immune system is involved in the pathogenesis, future research should also focus on the possibility of biological profiling in order to identify women at an increased risk of capsular contraction and therefore offer them the best possible treatment with the lowest possible risk.

\section{REFERENCES}

1. Johnson M. Breast implants: history, safety, and imaging. Radiol Technol 2013;84:439M-515M.

2. Namnoum JD, Largent J, Kaplan HM, et al. Primary breast augmentation clinical trial outcomes stratified by surgical incision, anatomical placement and implant device type. J Plast Reconstr Aesthet Surg 2013;66:1165-72.

3. Siggelkow W, Faridi A, Spiritus K, et al. Histological analysis of silicone breast implant capsules and correlation with capsular contracture. Biomaterials 2003;24:1101-9.

4. Daniels AU. Silicone breast implant materials. Swiss Med Wkly 2012;142:w13614.

5. Handel N, Cordray T, Gutierrez J, et al. A long-term study of outcomes, complications, and patient satisfaction with breast implants. Plast Reconstr Surg 2006;117:757-67.

6. Wolfram D, Rainer C, Niederegger $\mathrm{H}$, et al. Cellular and molecular composition of fibrous capsules formed around silicone breast implants with special focus on local immune reactions. J Autoimmun 2004;23:81-91.

7. Prantl L, Poppl N, Horvat N, et al. Serologic and histologic findings in patients with capsular contracture after breast augmentation with smooth silicone gel implants: is serum hyaluronan a potential predictor? Aesthetic Plast Surg 2005;29: 510-8.

8. Spear SL, Baker JL Jr. Classification of capsular contracture after prosthetic breast reconstruction. Plast Reconstr Surg 1995;96:1119-23.

9. Spear SL, Murphy DK; Allergan Silicone Breast Implant U. S. Core Clinical Study Group. Natrelle round silicone breast implants: Core Study results at 10 years. Plast Reconstr Surg 2014;133:1354-61.

10. Blount AL, Martin MD, Lineberry KD, et al. Capsular contracture rate in a low-risk population after primary augmentation mammaplasty. Aesthet Surg J 2013;33:516-21.

11. Stutman RL, Codner M, Mahoney A, et al. Comparison of breast augmentation incisions and common complications. Aesthetic Plast Surg 2012;36:1096-104.

12. Codner MA, Mejia JD, Locke MB, et al. A 15-year experience with primary breast augmentation. Plast Reconstr Surg 2011; 127:1300-10.

13. Sevin A, Sevin K, Senen D, et al. Augmentation mammaplasty: retrospective analysis of 210 cases. Aesthetic Plast Surg 2006;30:651-4.

14. Gutowski KA, Mesna GT, Cunningham BL. Saline-filled 
breast implants: a Plastic Surgery Educational Foundation multicenter outcomes study. Plast Reconstr Surg 1997;100: 1019-27.

15. Puckett CL. On the safety of silicone gel breast implants. Cancer Invest 2000;18:285.

16. Steiert AE, Boyce M, Sorg H. Capsular contracture by silicone breast implants: possible causes, biocompatibility, and prophylactic strategies. Med Devices (Auckl) 2013;6:211-8.

17. Tan KT, Wijeratne D, Shih B, et al. Tumour necrosis factoralpha expression is associated with increased severity of periprosthetic breast capsular contracture. Eur Surg Res 2010; 45:327-32.

18. Prantl L, Angele P, Schreml S, et al. Determination of serum fibrosis indexes in patients with capsular contracture after augmentation with smooth silicone gel implants. Plast Reconstr Surg 2006; 118:224-9.

19. Brazin J, Malliaris S, Groh B, et al. Mast cells in the periprosthetic breast capsule. Aesthetic Plast Surg 2014;38:592-601.

20. Moyer KE, Ehrlich HP. Capsular contracture after breast reconstruction: collagen fiber orientation and organization. Plast Reconstr Surg 2013;131:680-5.

21. Persichetti P, Segreto F, Carotti S, et al. Oestrogen receptoralpha and -beta expression in breast implant capsules: experimental findings and clinical correlates. J Plast Reconstr Aesthet Surg 2014;67:308-15.

22. Wolfram D, Rabensteiner E, Grundtman C, et al. T regulatory cells and TH17 cells in peri-silicone implant capsular fibrosis. Plast Reconstr Surg 2012;129:327e-337e.

23. Momoh AO, Chung KC. Discussion. T regulatory cells and TH17 cells in peri-silicone implant capsular fibrosis. Plast Reconstr Surg 2012;129:338e-339e.

24. Smalley DL, Shanklin DR, Hall MF. Monocyte-dependent stimulation of human $T$ cells by silicon dioxide. Pathobiology 1998;66:302-5.

25. Ojo-Amaize EA, Conte V, Lin HC, et al. Silicone-specific blood lymphocyte response in women with silicone breast implants. Clin Diagn Lab Immunol 1994;1:689-95.

26. Ulrich D, Lichtenegger F, Eblenkamp M, et al. Matrix metalloproteinases, tissue inhibitors of metalloproteinases, aminoterminal propeptide of procollagen type III, and hyaluronan in sera and tissue of patients with capsular contracture after augmentation with Trilucent breast implants. Plast Reconstr Surg 2004; 114:229-36.

27. Katzel EB, Koltz PF, Tierney R, et al. The impact of Smad3 loss of function on TGF-beta signaling and radiation-induced capsular contracture. Plast Reconstr Surg 2011;127:2263-9.

28. Kuhn MA, Wang X, Payne WG, et al. Tamoxifen decreases fibroblast function and downregulates TGF(beta2) in du- puytren's affected palmar fascia. J Surg Res 2002;103:14652.

29. Mikulec AA, Hanasono MM, Lum J, et al. Effect of tamoxifen on transforming growth factor beta1 production by keloid and fetal fibroblasts. Arch Facial Plast Surg 2001;3:111-4.

30. Marques M, Brown SA, Cordeiro ND, et al. Effects of fibrin, thrombin, and blood on breast capsule formation in a preclinical model. Aesthet Surg J 2011;31:302-9.

31. Kyle DJ, Harvey AG, Shih B, et al. Identification of molecular phenotypic descriptors of breast capsular contracture formation using informatics analysis of the whole genome transcriptome. Wound Repair Regen 2013;21:762-9.

32. Wolfram D, Oberreiter B, Mayerl C, et al. Altered systemic serologic parameters in patients with silicone mammary implants. Immunol Lett 2008; 118:96-100.

33. Marques M, Brown SA, Cordeiro ND, et al. Effects of coagulase-negative staphylococci and fibrin on breast capsule formation in a rabbit model. Aesthet Surg J 2011;31:420-8.

34. Tamboto H, Vickery K, Deva AK. Subclinical (biofilm) infection causes capsular contracture in a porcine model following augmentation mammaplasty. Plast Reconstr Surg 2010;126:835-42.

35. Drinane JJ, Bergman RS, Folkers BL, et al. Revisiting Triple Antibiotic Irrigation of Breast Implant Pockets: a Placebocontrolled Single Practice Cohort Study. Plast Reconstr Surg Glob Open 2013;1:e55.

36. Spear SL, Jespersen MR. Breast implants: saline or silicone? Aesthet Surg J 2010;30:557-70.

37. Bengtson BP, Van Natta BW, Murphy DK, et al. Style 410 highly cohesive silicone breast implant core study results at 3 years. Plast Reconstr Surg 2007;120:40S-48S.

38. Maxwell GP, Van Natta BW, Murphy DK, et al. Natrelle style 410 form-stable silicone breast implants: core study results at 6 years. Aesthet Surg J 2012;32:709-17.

39. Heden P, Bone B, Murphy DK, et al. Style 410 cohesive silicone breast implants: safety and effectiveness at 5 to 9 years after implantation. Plast Reconstr Surg 2006; 118:1281-7.

40. Cunningham B. The Mentor Core Study on Silicone MemoryGel Breast Implants. Plast Reconstr Surg 2007;120:19S29S.

41. Hammond DC, Migliori MM, Caplin DA, et al. Mentor Contour Profile Gel implants: clinical outcomes at 6 years. Plast Reconstr Surg 2012;129:1381-91.

42. Walker PS, Walls B, Murphy DK. Natrelle saline-filled breast implants: a prospective 10-year study. Aesthet Surg J 2009; 29:19-25.

43. El-Sheikh Y, Tutino R, Knight C, et al. Incidence of capsular contracture in silicone versus saline cosmetic augmentation 
mammoplasty: a meta-analysis. Can J Plast Surg 2008;16: 211-5.

44. Barnsley GP, Sigurdson LJ, Barnsley SE. Textured surface breast implants in the prevention of capsular contracture among breast augmentation patients: a meta-analysis of randomized controlled trials. Plast Reconstr Surg 2006;117: 2182-90.

45. Wong CH, Samuel M, Tan BK, et al. Capsular contracture in subglandular breast augmentation with textured versus smooth breast implants: a systematic review. Plast Reconstr Surg 2006;118:1224-36.

46. Hakelius L, Ohlsen L. A clinical comparison of the tendency to capsular contracture between smooth and textured gelfilled silicone mammary implants. Plast Reconstr Surg 1992; 90:247-54.

47. Schaub TA, Ahmad J, Rohrich RJ. Capsular contracture with breast implants in the cosmetic patient: saline versus silicone-a systematic review of the literature. Plast Reconstr Surg 2010; 126:2140-9.

48. Poeppl N, Schreml S, Lichtenegger F, et al. Does the surface structure of implants have an impact on the formation of a capsular contracture? Aesthetic Plast Surg 2007;31:133-9.

49. Stevens WG, Nahabedian MY, Calobrace MB, et al. Risk factor analysis for capsular contracture: a 5-year Sientra study analysis using round, smooth, and textured implants for breast augmentation. Plast Reconstr Surg 2013;132:1115-23.

50. Abramo AC, De Oliveira VR, Ledo-Silva MC, et al. How texture-inducing contraction vectors affect the fibrous capsule shrinkage around breasts implants? Aesthetic Plast Surg 2010;34:555-60.

51. Jacombs A, Tahir S, Hu H, et al. In vitro and in vivo investigation of the influence of implant surface on the formation of bacterial biofilm in mammary implants. Plast Reconstr Surg 2014;133:471e-480e.

52. Minami E, Koh IH, Ferreira JC, et al. The composition and behavior of capsules around smooth and textured breast implants in pigs. Plast Reconstr Surg 2006;118:874-84.

53. Hall-Findlay EJ. Breast implant complication review: double capsules and late seromas. Plast Reconstr Surg 2011;127:5666.

54. Pompei S, Arelli F, Labardi L, et al. Breast reconstruction with polyurethane implants: preliminary report. Eur J Plast Surg 2012;35:441-7.

55. Vazquez G, Pellon A. Polyurethane-coated silicone gel breast implants used for 18 years. Aesthetic Plast Surg 2007;31:330-6.

56. Bucky LP, Ehrlich HP, Sohoni S, et al. The capsule quality of saline-filled smooth silicone, textured silicone, and polyurethane implants in rabbits: a long-term study. Plast Reconstr
Surg 1994;93:1123-31.

57. Henriksen TF, Fryzek JP, Holmich LR, et al. Surgical intervention and capsular contracture after breast augmentation: a prospective study of risk factors. Ann Plast Surg 2005;54: 343-51.

58. Pereira Leite L, Correia Sa I, Marques M. Etiopathogenesis and treatment of breast capsular contracture. Acta Med Port 2013;26:737-45.

59. Cunningham B, McCue J. Safety and effectiveness of Mentor's MemoryGel implants at 6 years. Aesthetic Plast Surg 2009;33:440-4.

60. Little G, Baker JL Jr. Results of closed compression capsulotomy for treatment of contracted breast implant capsules. Plast Reconstr Surg 1980;65:30-3.

61. Forster NA, Kunzi W, Giovanoli P. The reoperation cascade after breast augmentation with implants: what the patient needs to know. J Plast Reconstr Aesthet Surg 2013;66:31322.

62. Adams WP Jr. Capsular contracture: what is it? What causes it? How can it be prevented and managed? Clin Plast Surg 2009;36:119-26.

63. Lee HK, Jin US, Lee YH. Subpectoral and precapsular implant repositioning technique: correction of capsular contracture and implant malposition. Aesthetic Plast Surg 2011; 35:1126-32.

64. Maxwell GP, Gabriel A. The neopectoral pocket in revisionary breast surgery. Aesthet Surg J 2008;28:463-7.

65. Maxwell GP, Birchenough SA, Gabriel A. Efficacy of neopectoral pocket in revisionary breast surgery. Aesthet Surg J 2009; 29:379-85.

66. Castello MF, Lazzeri D, Silvestri A, et al. Maximizing the use of precapsular space and the choice of implant type in breast augmentation mammaplasty revisions: review of 49 consecutive procedures and patient satisfaction assessment. Aesthetic Plast Surg 2011;35:828-38.

67. Salgarello M, Visconti G, Farallo E. Autologous fat graft in radiated tissue prior to alloplastic reconstruction of the breast: report of two cases. Aesthetic Plast Surg 2010;34:5-10.

68. Sarfati I, Ihrai T, Kaufman G, et al. Adipose-tissue grafting to the post-mastectomy irradiated chest wall: preparing the ground for implant reconstruction.J Plast Reconstr Aesthet Surg 2011;64:1161-6.

69. Roca GB, Graf R, da Silva Freitas R, et al. Autologous fat grafting for treatment of breast implant capsular contracture: a study in pigs. Aesthet Surg J 2014;34:769-75.

70. Cheng A, Lakhiani C, Saint-Cyr M. Treatment of capsular contracture using complete implant coverage by acellular dermal matrix: a novel technique. Plast Reconstr Surg 2013;132: 
519-29.

71. Schmitz M, Bertram M, Kneser U, et al. Experimental total wrapping of breast implants with acellular dermal matrix: a preventive tool against capsular contracture in breast surgery? J Plast Reconstr Aesthet Surg 2013;66:1382-9.

72. Kornstein A. Porcine-derived acellular dermal matrix in primary augmentation mammoplasty to minimize implant-related complications and achieve an internal mastopexy: a case series. J Med Case Rep 2013;7:275.

73. Hester TR Jr, Ghazi BH, Moyer HR, et al. Use of dermal matrix to prevent capsular contracture in aesthetic breast surgery. Plast Reconstr Surg 2012;130:126S-136S.

74. Hille-Betz U, Kniebusch N, Wojcinski S, et al. Breast reconstruction and revision surgery for implant-associated breast deformities using porcine acellular dermal matrix: a multicenter study of 156 cases. Ann Surg Oncol 2015;22:114652.

75. Bastos EM, Sabino Neto M, Garcia EB, et al. Effect of zafirlukast on capsular contracture around silicone implants in rats. Acta Cir Bras 2012;27:1-6.

76. Spano A, Palmieri B, Taidelli TP, et al. Reduction of capsular thickness around silicone breast implants by zafirlukast in rats. Eur Surg Res 2008;41:8-14.

77. Reid RR, Greve SD, Casas LA. The effect of zafirlukast (Accolate) on early capsular contracture in the primary augmentation patient: a pilot study. Aesthet Surg J 2005;25:26-30.

78. Schlesinger SL, Ellenbogen R, Desvigne MN, et al. Zafirlukast (Accolate): a new treatment for capsular contracture. Aesthet Surg J 2002;22:329-36.

79. Scuderi N, Mazzocchi M, Fioramonti P, et al. The effects of zafirlukast on capsular contracture: preliminary report. Aesthetic Plast Surg 2006;30:513-20.

80. Scuderi N, Mazzocchi M, Rubino C. Effects of zafirlukast on capsular contracture: controlled study measuring the mammary compliance. Int J Immunopathol Pharmacol 2007;20: 577-84.
81. Mazzocchi M, Dessy LA, Alfano C, et al. Effects of zafirlukast on capsular contracture: long-term results. Int J Immunopathol Pharmacol 2012;25:935-44.

82. Kim YS, Hong JW, Yoon JH, et al. Botulinum toxin a affects early capsule formation around silicone implants in a rat model. Ann Plast Surg 2015;74:488-95.

83. Lee SD, Yi MH, Kim DW, et al. The effect of botulinum neurotoxin type $\mathrm{A}$ on capsule formation around silicone implants: the in vivo and in vitro study. Int Wound J 2014 Mar 7 [Epub]. http://dx.doi.org/10.1111/iwj.12228.

84. Lee SG, Lee SD, Kim MK, et al. Effect of antiadhesion barrier solution and fibrin on capsular formation after silicone implant insertion in a white rat model. Aesthetic Plast Surg 2015;39:162-70.

85. Lew DH, Yoon JH, Hong JW, et al. Efficacy of antiadhesion barrier solution on periimplant capsule formation in a white rat model. Ann Plast Surg 2010;65:254-8.

86. Shin KC, Chung KI, Park BY, et al. The effect of antiadhesion agent on peri-implant capsular formation in rabbits. Ann Plast Surg 2013;71:600-4.

87. Ye X, Shokrollahi K, Rozen WM, et al. Anaplastic large cell lymphoma (ALCL) and breast implants: breaking down the evidence. Mutat Res Rev Mutat Res 2014;762:123-32.

88. Miranda RN, Aladily TN, Prince HM, et al. Breast implantassociated anaplastic large-cell lymphoma: long-term followup of 60 patients. J Clin Oncol 2014;32:114-20.

89. Peters W. Update on anaplastic large cell lymphoma in women with breast implants. Can J Plast Surg 2014;22:267-9.

90. Thompson PA, Prince HM. Breast implant-associated anaplastic large cell lymphoma: a systematic review of the literature and mini-meta analysis. Curr Hematol Malig Rep 2013; 8:196-210.

91. Lazzeri D, Zhang YX, Huemer GM, et al. Capsular contracture as a further presenting symptom of implant-related anaplastic large cell lymphoma. Am J Surg Pathol 2012;36:1735-6. 